\title{
POLARIZED TARGETS AND BEAMS-PARALLEL SESSION SUMMARY
}

\author{
J. P. CHEN \\ Jefferson Lab, Newport News, VA23606, USA \\ E-mail: jpchen@jlab.org
}

\begin{abstract}
Recent progress on the polarized targets and polarized beams at Bonn, Brookhaven, Jefferson Lab, Mainz and PSI were reported in the parallel sessions. Summary of the progress is presented here.
\end{abstract}

\section{Introduction}

Progress in polarized targets and polarized beams made possible the study of the nucleon spin structure and the GDH sum rule. A parallel session on polarized targets and another on polarized beams were organized to present and discuss the recent progress and development from the laboratories involved in the nucleon spin structure and GDH studies.

In the parallel session on the polarized targets, D. Crabb ( Virginia) reported the progress at Jefferson Lab on the polarized solid targets for electron scattering experiments ${ }^{1}$. W. Meyer (Bochum) presented the progress of frozen spin polarized solid targets at Bonn and Mainz ${ }^{2}$. B. van den Brandt (PSI) showed the progress of the polarized scintillating targets at $\mathrm{PSI}^{3}$. S. Whisnant (South Carolina) reported the progress of the polarized HD ice target at LEGS $^{4}$. W. Heil (Mainz) presented the progress of the polarized ${ }^{3} \mathrm{He}$ target at $\mathrm{MAMI}^{5}$ and I. Kominis (Princeton) showed the progress of the spin exchange polarized ${ }^{3}$ He target at $\mathrm{JLab}^{6}$.

In the parallel session on the polarized beams, C. Sinclair (JLab) presented the progress of the polarized source at $\mathrm{JLab}^{7}$. K. Aulenbacher (Mainz) showed the progress of the polarized electron beam at $\mathrm{MAMI}^{8}$. W. Hillert (Bonn) reported on a new injector for polarized electrons at ELSA ${ }^{9}$.

It is evident from the presentations that the progresses in the last decade are very impressive. Technologies in both polarized targets and beams are becoming mature. New developments and improvements are still emerging to meet the increasing demand of this field. Stimulating discussions followed after the presentations.

targbeam: submitted to World Scientific on August 30, 2000 


\section{Polarized Targets}

\subsection{General Principle}

When a target is under an external magnetic field (B) at a certain temperature $(\mathrm{T})$, the energy split of the different particle spin states depends on $\mu \mathrm{B} / \mathrm{kT}$, where $\mu$ is the magnetic moment of the particle under consideration. The population of particles at different spin states follows the Boltzmann distribution when they reach an equilibrium state: the lower energy spin state will have more population than the higher energy spin state. Therefore the target is polarized under the magnetic field (so called brute-force polarization). Since the magnetic moment of a nucleon or a nucleus is very small, it takes a very high magnetic field and a very low temperature to be able to reach a significant polarization. For example, the LEGS ice HD target is usually polarized at 17 Tesla field and $15 \mathrm{mK}$ temperature.

Since an electron has a much larger magnetic moment than a nucleon or a nucleus, it is much easier to polarize electrons in atoms than to polarize nucleons or nuclei in the atoms. Taking advantage of this fact, the Dynamical Nuclear Polarization (DNP) method has been widely used in polarized solid targets. Optical pumping and spin exchange technique has been used to polarize gaseous ${ }^{3} \mathrm{He}$ target.

For a polarized target to be useful in experiments, the polarization rate needs to be much larger than the relaxation (de-polarization) rate. The interaction of the polarizing nuclei or atoms with the environment are crucial in determining the polarization rate and the relaxation rate. This has been a very important factor in choosing the target material.

From experimental consideration, figure of merit is often used to quickly comparing different methods for experimental instruments. In the case of polarized targets, figure of merit could be defined as following: $F=n_{t} P_{t}^{2} f^{2}$, where $n_{t}$ is the number of target nuclei, $P_{t}$ is the target polarization and $f$ is the dilution factor - the ratio of the contribution from the desired material to the total material. Therefore it depends on the reaction under consideration. One defination, which is independent of the reaction, often used for easy comparison, is the ratio by weight of desired material to the total material. (Another factor, which is also important for electron scattering but not included in the above FOM definition, is the amount of beam the target can withstand). Large efforts have been put in the last decade to optimize the target polarization, increase the density of the target and increase the dilution factor.

targbeam: submitted to World Scientific on August 30, 2000 


\subsection{Dynamical Nuclear Polarization: Polarized Solid targets}

The principle of DNP can be illustrated using an example of polarized proton target in $\mathrm{NH}_{3}$. At 5 Tesla and $1 \mathrm{~K}$ condition, protons have little polarization while the atomic electrons are nearly fully polarized. The difference in energy levels for the two spin states for electrons is about $140 \mathrm{GHz}$, corresponding to microwave frequency. Exact frequency microwave is used to pump the target from the lower electron spin state and a specific proton spin state to the higher electron spin state (opposite proton spin state). It will spontaneously decay back to the lower electron spin state, with proton spin being either one of the two states. Since the population in one proton spin state is continuously being pumped away, eventually most particles will end up being at the other proton spin state, i. e. the proton is highly polarized. The DNP method is widely used in polarized solid targets.

D. Crabb presented the status of the solid polarized targets used at Jefferson Lab Hall C (same one also used at SLAC) and Hall B. Three target materials have been used: $\mathrm{NH}_{3}, \mathrm{ND}_{3}$ and ${ }^{6} \mathrm{LiD}$. All of these targets have reasonable radiation resistance: can withstand $100 \mathrm{nA}$ electron beam. Polarization for proton reached above $90 \%$. While for deuteron, it reached $40 \%$ with $\mathrm{ND}_{3}$. With ${ }^{6} \mathrm{LiD}$, the polarization achieved is less than $30 \%$. The dilution factor is 0.18 for $\mathrm{NH}_{3}$ and 0.3 for $\mathrm{ND}_{3}$. The main reason to use ${ }^{6} \mathrm{LiD}$ is to improve the dilution factor: since ${ }^{6} \mathrm{Li}$ is approximately an $\alpha$ particle plus a deuteron, the dilution factor for ${ }^{6} \mathrm{LiD}$ is approximately 0.5 . The luminosity for the polarized solid target can reach up to $10^{35}$ particles $/ \mathrm{sec}$. Polarization is usually measured with NMR. The precision reached is $2.5 \%$ for the proton and $4 \%$ for the deuteron.

Possible future development of a polarized tritium target was briefly discussed. It is clearly of great interest to have such a polarized target. D. Crabb described a possible polarized solid ${ }^{7} \mathrm{LiT}$ target. It is expected to be similar to polarized Hydrogen target: polarization should reach $60-70 \% .{ }^{7} \mathrm{Li}$ is chosen to maximize the dilution factor (same as ${ }^{6} \mathrm{Li}$ is in ${ }^{6} \mathrm{LiD}$ ). Safety and some other issues have been considered and further study will be needed. Some $\mathrm{R} \& \mathrm{D}$ effort will take place soon.

\subsection{Frozen Spin Butanol and Deuterized Butanol Targets}

After a general introduction and a review of progress of the polarized solid targets for both real and virtual photon scattering, W. Meyer concentrated his talk on the frozen spin polarized Butanol $\left(\mathrm{C}_{4} \mathrm{H}_{9} \mathrm{OH}\right)$ and Deuterized Butanol $\left(C_{4} D_{9} O D\right)$ targets, which are being used at Mainz and Bonn for GDH sum rule experiments. The target is polarized with field of $2.5 \mathrm{~T}$, temperature of

targbeam: submitted to World Scientific on August 30, 2000 
about $300 \mathrm{mK}$ and microwave pumping. Temperature of $50 \mathrm{mK}$ and a field of about $0.35 \mathrm{~T}$ are used to keep the spin frozen when it is used in photon beam. A special thin-wall solenoid magnet provides the in-beam holding field while having little obstruction to the scattered particles. The polarization can reach up to $90 \%$ for the proton and $50 \%$ for the deuteron. The advantage of the polarized Butanol target is that the unwanted nuclei $(\mathrm{C}$ and $\mathrm{O})$ are unpolarized. The limitation is that it can only be used for low intensity beam, such as photon beam or muon beam. The dilution factor is 0.13 for butanol and 0.23 for Deuterized Butanol. The polarization measurement using NMR reached a precision of about $2 \%$ for the proton.

\subsection{Polarized Scintillating Targets at PSI}

B. van den Brandt described the progress in the development of a new type of polarized targets: polarized scintillating targets. Since the nuclei in the scintillating detector itself are being polarized, it is possible to measure very low momentum particles in target. Therefore a wealth of new possibilities are opened up. The polarizing material is usually some type of polymer. The nuclei that could be polarized are proton, deuteron, ${ }^{6} \mathrm{Li},{ }^{7} \mathrm{Li},{ }^{15} \mathrm{~N}$ and ... . Polarization can be reasonably high (above $60 \%$ for proton). Since scintillating material is usually double bounding, polarization requires doping with non-double bounding material to have free radicals. TEMPO is one of the materials used for doping. Since the target is at high magnetic field and low temperature, it is a challenge to get the scintillating light out to PMTs. Several different diameters light guide have been tested. The biggest one is $19 \mathrm{~mm}$. The latest results are reported in a NIM article ${ }^{10}$.

\subsection{The Polarized HD Ice Target at LEGS}

S. Whisnant reported the progress of the polarized ice HD target at LEGS. The ice HD is polarized using the brute-force method: large field of $17 \mathrm{~T}$ and very low temperature of $10-15 \mathrm{mK}$. Since the coupling of pure HD spins to the lattice is very weak, small amount (a few parts in $10^{4}$ ) of ortho- $\mathrm{H}_{2}$ $\left(\mathrm{o}-\mathrm{H}_{2}\right)$ is added to have adequate spin-lattice coupling to polarize the HD target. However, $\mathrm{o}-\mathrm{H}_{2}$ also makes the relaxation time short: the target will depolarize easily. Luckily, nature provides us with a special relaxation switch: $\mathrm{o}-\mathrm{H}_{2}$ decays to $\mathrm{p}-\mathrm{H}_{2}$ (para- $\mathrm{H}_{2}$ ) with a 6.25 days mean life and $\mathrm{p}-\mathrm{H}_{2}$ has very weak coupling to the spin-lattice. Therefore after most of the $\mathrm{o}-\mathrm{H}_{2}$ molecules decay to $\mathrm{p}-\mathrm{H}_{2}$, the relaxation time becomes very long. It usually takes over one month to polarize and freeze the polarization. Once the target polarization has been frozen, it is stored in a $10 \mathrm{~T}$ field at a temperature of $1.5 \mathrm{~K}$. The

targbeam: submitted to World Scientific on August 30, 2000 
target polarization life time will be 3 months for $\mathrm{H}$ and 8 months for $\mathrm{D}$ in the storage condition. When doing experiment, the target will be transfered to an in-beam field of $0.75 \mathrm{~T}$ at temperature of $1.5 \mathrm{~K}$, in which condition the $\mathrm{H}$ polarization will have a life time of 2 weeks and $\mathrm{D}$ polarization 6 weeks.

With $17 \mathrm{~T}$ and $15 \mathrm{mK}$, the proton polarization can reach $81 \%$ while the deuteron polarization can only reach $23 \%$. To get high deuteron polarization, Adiabatic Fast Passage can be used to induce first forbidden RF transition to transfer $\mathrm{H}$ polarization to $\mathrm{D}$.

When the $\mathrm{o}-\mathrm{H}_{2}$ decays to $\mathrm{p}-\mathrm{H}_{2}$, small amount of heat will be released. To get the heat out of the ice HD target, about $10 \%$ of thin aluminum wires have to be embedded into the ice HD target.

The clear advantage of the HD target is its high dilution factor $(0.28$ for $\mathrm{H}$ and 0.55 for $\mathrm{D}$ ) and the reduced background.

As it often is the case for new technologies, a lot of difficult problems need to be overcome. After over thirty years study and experimenting, the first polarized HD (with $\mathrm{H}$ polarized) was finally produced last year. The first beam test is planned this year. This innovative target will open up a new avenue for experiments to study the spin structure of the nucleons.

\subsection{The polarized ${ }^{3}$ He target at MAMI}

W. Heil presented the status of the polarized ${ }^{3} \mathrm{He}$ target at MAMI. Two methods are widely used to polarize ${ }^{3} \mathrm{He}$ : 1) spin exchange with optically pumped Rb-vapor, and 2) optical pumping of metastable ${ }^{3} \mathrm{He}^{*}$ atoms. The MAMI polarized ${ }^{3} \mathrm{He}$ target uses the second method.

Metastable ${ }^{3} S_{1}$ atoms are produced by an electrodeless weak RF discharge in a glass cell filled with pure ${ }^{3} \mathrm{He}$. The cell is placed in a weak uniform magnetic field. Right-handed (or left-handed) circularly polarized laser light at $1083.4 \mathrm{~nm}$, corresponding to the transition of ${ }^{3} S_{1} \rightarrow^{3} P_{0}$, excites the transition from ${ }^{3} S_{1}$ to ${ }^{3} P_{0}$ with the selection rule $\Delta m=+1($ or -1$)$. The pumping light excites atoms from the $M_{F}=-1 / 2$ and $m_{F}=-3 / 2$ sublevels of the metastable state to the ${ }^{3} P_{0}$ level which then decay back to all sublevels of ${ }^{3} S_{1}$ through spontaneous emission. The result is that atoms from $m_{F}=-1 / 2$ and $-3 / 2$ are transferred to $+1 / 2$ and $+3 / 2$. Therefore the metastable atoms become polarized. The hyperfine interaction mixes the electron polarization with the nuclear polarization. The polarization of the metastable atoms is transferred to the ground state through metastability-exchange collision. Since the ground state ${ }^{3} \mathrm{He}$ is in $\mathrm{J}=0$ state, the atomic polarization means the nucleus is also polarized.

It is usually difficult to have high density with this method because the

targbeam: submitted to World Scientific on August 30, 2000 
${ }^{3} \mathrm{He}$ density has to be low to maintain the RF discharge. The MAMI ${ }^{3} \mathrm{He}$ target uses a special compression technique to reach pressures up to 10 bars (the highest with the metastable optical pumping method). Polarization can reach up to $50 \%$. The depolarization without beam is due to the intrinsic dipolar interaction, the wall effect and the field inhomogeneity. The relaxation time due to the dipolar interaction is $750 / p($ barh $)$. The wall effect is about $570 \mathrm{~h}$. The field inhomogeneity is usually very small. For high pressure cell, the dipolar is the dominate effect.

Relaxation under electron beam condition are due to 1) ionization, 2) catalytic relaxation by ${ }^{3} \mathrm{He}_{2}^{+}$molecules and 3) creation of paramagnetic centers in the glass. For the MAMI target of $20 \mathrm{~cm}$ length at 4 bars with volume of $300 \mathrm{cc}$ under $10 \mu \mathrm{A}$ beam, the relaxation times for the three factors are 150 $\mathrm{h}, 150 \mathrm{~h}$ and $30-35 \mathrm{~h}$ respectively.

The MAMI polarized ${ }^{3} \mathrm{He}$ target is being used for MRI, neutron spin filter, $G_{e n}$ and ${ }^{3} \mathrm{He}$ nuclear physics study. It is also planned for GDH study.

\subsection{The Spin-Exchange Polarized ${ }^{3}$ He Target at JLab}

I. Kominis presented the polarized ${ }^{3} \mathrm{He}$ target at Jefferson Lab, which uses the method of spin-exchange with polarized $\mathrm{Rb}$. $\mathrm{Rb}$ vapor is polarized by optical pumping with circularly polarized $795 \mathrm{~nm}$ laser light. Then the Rb electron polarization is transferred to the ${ }^{3} \mathrm{He}$ nucleus by spin-exchange hyperfine interaction.

The JLab target has two sets of holding coils, which enable polarization in either longitudinal or transverse direction (or any direction in the scattering plane). Two sets of three 30 Watts diode lasers provide ample power for optical pumping. The target has a double-cell system: a spherical shaped pumping cell connected with a $40 \mathrm{~cm}$ long cylindrical target cell. Several target cells were used for the first two experiments at the end of 1998 and beginning of 1999, all have densities of more than 10 amagats (1 amagat corresponds to gas density at 1 atomsphere and $0{ }^{\circ} \mathrm{C}$ ). The target polarization without beam reached up to $50 \%$. The depolarization is dominated by the dipolar interaction and the cell wall effect. With the high density, the dipolar interaction gives a relaxation time constant of about $80 \mathrm{~h}$, and the relaxation time constant for the wall effect is larger than $80 \mathrm{~h}$. Under electron beam up to $15 \mu \mathrm{A}$, the average polarization achieved is about $35 \%$. The relaxation time is about $30 \mathrm{~h}$ at a beam current of $10 \mu \mathrm{A}$.

The target polarization was measured with two methods: NMR with Adiabatic Fast Passage (AFP), calibrated with water signal, and EPR (ElectronMagnetic Resonance), which measures the $\mathrm{Rb}$ energy level shift due to the

targbeam: submitted to World Scientific on August 30, 2000 
small field induced by the ${ }^{3} \mathrm{He}$ polarization. The two methods agreed within the uncertainty (of about $4 \%$ ). The polarimetry was also checked with elastic scattering asymmetry: the ratio of the measured asymmetry and the calculated asymmetry from the world elastic from factor data is proportional to the product of the target and the beam polarization. Since the beam polarization is measured with a Møller polarimeter with a precision of $3 \%$, the elastic asymmetry gives a good check of the target polarimetry. They agreed well within the measurement uncertainties.

\section{Polarized Beams}

\subsection{General Principle}

The most commonly used source for polarized electrons is photoemission from GaAs. However for bulk GaAs crystal, due to the degeneracy of the highest valence band, the maximum polarization one can reach is only $50 \%$. Strained GaAs has been recently used in many laboratories to reach high polarization. With Strained GaAs, the degeneracy is removed, so the maximum polarization can be $100 \%$, although in practice it usually reaches about $80 \%$. Other methods can also be used to remove the degeneracy: strained layer super-lattice crystal, or natural no-degeneracy crystal.

The figure of merit for a polarized beam is $F=I P^{2}$, where $\mathrm{I}$ is the beam current (intensity) and $\mathrm{P}$ is the polarization. Reaching highest polarization with high intensity has been a challenge for a long time. The progress in the last a few years at Jefferson Lab, Mainz and Bonn has been very impressive. It has been one of the main reasons for the success in the field of nucleon spin structure study. For some experiments, for example the real photon GDH experiment, the beam current is limited by how much the target or the detector can withstand. In this case then only the polarization goes into the figure of merit.

\subsection{The Jefferson Lab Polarized Electron Source}

After giving some historical account, C. Sinclair reported the progress of the polarized electron source at Jefferson Lab. With three experimental halls running experiments simultaneously, the source needs continuous high current operation. Polarization, as well as quantum efficiency and life time are all of important consideration. Ultrahigh vacuum is essential for high quantum efficiency and long life time. The crystal is usually coated with Cs. To increase the life time of a cathode, re-cesiation and thermal annealing were used to restore performance of the cathode. With improvement in the high vacuum

targbeam: submitted to World Scientific on August 30, 2000 
and reasonable laser power, the polarized source was able to deliver more than $50 \mu \mathrm{A} 80 \%$ polarized electron beam with long life time (over 100 hours). The maximum current reached over $100 \mu \mathrm{A}$ when combining three lasers together. More powerful new lasers are planned to be installed to reach even higher beam intensity.

With a Wien filter, any polarization direction can be chosen. Usually one tries to have the maximize longitudinal polarization on the target. However, with 3-hall operation, only at special 'magic' beam energies, it is possible to get all three halls to have $100 \%$ of the source polarization. In most of the other energies, the Wien Filter is set to optimize the product of the three hall's longitudinal polarization, which usually can reach above $90 \%$ of the source polarization.

\subsection{The Polarized Electron Beam at MAMI}

K. Aulenbacher presented the status of the polarized electron beam at MAMI. The source also uses the strained GaAs. The maximum polarization usually reaches about $80 \%$. Due to the limited space for the current set-up, no Wien Filter was installed. Again the maximum longitudinal polarization can be reached only at a set of special beam energies. Tow of the special energies were used for the first GDH experiment.

K. Aulenbacher also discussed the study and outlook to improve the beam polarization. The main limitation is the incomplete polarization in the strained GaAs crystal. Other factors, such as extraction of polarized electrons from the source, have shown to be not of big importance. Improvement in the source polarization to close to $90 \%$ maybe possible, but very difficult.

\subsection{A New Injector for Polarized Electrons at ELSA}

W. Hillert reported the progress of a new injector for polarized electrons at ELSA in Bonn. The source uses a strained layer superlattice crystal. The polarization at the source also reaches about $80 \%$. One of the main issue for ELSA is the low transport efficiency and low duty factor at the injector. Therefore it requires high power density and needs to face the challenge of overcoming polarization loss in transporting and accelerating. Another main issue is that there are a number of depolarizing resonances in ELSA. Big progress has been made to overcome the first few depolarizing resonances. The polarization remained above $70 \%$ at energy of $1.6 \mathrm{GeV}$, and above $40 \%$ at energy of $2.5 \mathrm{GeV}$. Effort is going on to overcome the depolarizing resonances between $1.6 \mathrm{GeV}$ to $3 \mathrm{GeV}$ for the GDH experiment, which is planned to start data taking soon.

targbeam: submitted to World Scientific on August 30, 2000 


\section{Acknowledgments}

I would like to thank Professor H.-J. Arends, the co-convenor for the polarized target and the polarized beam parallel seccions, for his help in organizing the sessions and making most of the arrangements, and for his careful proofreading of the manuscript. I would also like to thank Professor D. Drechsel and the local organizing committee for the well organized and successful symposium.

\section{References}

1. D. Crabb, presentation at this GDH Symposium, see the proceedings.

2. W. Meyer, presentation at this GDH Symposium, see the proceedings.

3. B. van den Brandt, presentation at this GDH Symposium, see the proceedings.

4. S. Whisnant, presentation at this GDH Symposium, see the proceedings.

5. W. Heil, presentation at this GDH Symposium, see the proceedings.

6. I. Kominis, presentation at this GDH Symposium, see the proceedings.

7. C. Sinclair, presentation at this GDH Symposium, see the proceedings.

8. K. Aulenbacher, presentation at this GDH Symposium, see the proceedings.

9. W. Hillert, presentation at this GDH Symposium, see the proceedings.

10. B. van den Brandt et al., Nucl. Inst. and Meth. A446, 592 (2000) 